\title{
The inverse effect of meal intake on controlled attenuation parameter and liver stiffness as assessed by transient elastography
}

Kanittha Ratchatasettakul ${ }^{1}$, Sasivimol Rattanasiri ${ }^{2}$, Kwannapa Promson ${ }^{1}$, Pranee Sringam ${ }^{1}$ and Abhasnee Sobhonslidsuk ${ }^{*}$ (D)

\begin{abstract}
Background: Controlled attenuation parameter (CAP) and liver stiffness (LS) measured by transient elastography $\left(T E\right.$, Fibroscan ${ }^{\oplus}$ ) have been used for steatosis and fibrosis assessment. We evaluated the effect of meal intake on CAP and LS values.

Methods: Forty patients who had had a liver biopsy within the previous month were recruited. The biopsy was graded for fibrosis (F) and steatosis (S) stagings. TE was performed after overnight fasting (baseline values) and 15, 30, 45, 60, 90, and 120 min following the intake of a standard commercial formula meal, and every 30 min until LS and CAP values returned to baseline. The effect of meal intake on CAP and LS values was analyzed with a multilevel mixed model approach.

Results: The mean age was $53.1 \pm 11.2$ years old. The mean (SD) BMI was $25.6 \pm 4.5 \mathrm{~kg} / \mathrm{m}^{2}$. F0, F1, F2, F3 and F4 fibrosis stages were found in 17 (42.5\%), 9 (22.5\%), 4 (10.0\%), 8 (20.0\%) and 2 (5.0\%), respectively. S0, S1, S2 and S3 steatosis stages were seen in $22(55.0 \%), 11(27.5 \%), 4(10.0 \%)$ and $3(7.5 \%)$, respectively. The mean (SD) CAP and median (IQR) LS values at baseline were $249.7 \pm 58.1 \mathrm{~dB} / \mathrm{m}$ and $11.9(6-18.1) \mathrm{kPa}$. A significant decrease in CAP values was observed in all patients 15 to 120 min after meals, with the CAP peak value at 60 min and the mean post-meal delta reduction of $18.1 \mathrm{~dB} / \mathrm{min}$. CAP values declined after meals at early fibrosis stages and across all stages of steatosis. A significant increase in LS values after meal intake was observed within 15 to 120 min, with the LS peak value at $15 \mathrm{~min}$ and the mean post-meal delta increase of $2.4 \mathrm{kPa}$. Post-meal CAP and LS values returned to baseline within 150 min following meals.

Conclusion: Following a meal, patients' CAP values declined with the peak value at 60 min, contrasting with the rising of LS values with the peak value at $15 \mathrm{~min}$. The post-meal CAP and LS values returned to baseline by 150 min. A fasting period of more than 150 min after a meal is recommended for patients undergoing TE.
\end{abstract}

Keywords: Controlled attenuation parameter, Liver stiffness, Transient elastography, Meal, Steatosis, Fibrosis

\footnotetext{
* Correspondence: abhasnee.sob@mahidol.ac.th

${ }^{1}$ Division of Gastroenterology and Hepatology, Department of Medicine,

Faculty of Medicine, Ramathibodi Hospital, Mahidol University, 270 Rama 6

Road, Bangkok 10400, Thailand

Full list of author information is available at the end of the article
}

(c) The Author(s). 2017 Open Access This article is distributed under the terms of the Creative Commons Attribution 4.0 International License (http://creativecommons.org/licenses/by/4.0/, which permits unrestricted use, distribution, and reproduction in any medium, provided you give appropriate credit to the original author(s) and the source, provide a link to the Creative Commons license, and indicate if changes were made. The Creative Commons Public Domain Dedication waiver (http://creativecommons.org/publicdomain/zero/1.0/) applies to the data made available in this article, unless otherwise stated. 


\section{Background}

Hepatic steatosis is a common feature in many types of chronic liver disease such as alcoholic liver disease, nonalcoholic fatty liver disease (NAFLD), and chronic viral hepatitis $B$ and $C[1,2]$. NAFLD is a frequent concomitant condition in chronic liver disease that can accelerate the progression of the disease [3]. NAFLD can reduce virological response in treatment of chronic hepatitis $\mathrm{C}$ [4]. Liver biopsy is a gold standard for the assessment of steatosis and other histological features [5]. However, this procedure is invasive and costly. Moreover, sampling errors and questionable reproducibility in liver biopsy have been reported [5-7].

A novel, non-invasive tool based on ultrasound attenuation, the controlled attenuation parameter (CAP), was developed to assess the degree of liver steatosis [8]. It is performed with transient elastrography (TE, FibroScan $\left.{ }^{\oplus}\right)$. The reliable diagnostic performance of CAP has been confirmed in many types of chronic liver disease, including chronic viral hepatitis and NAFLD [9, 10]. Previous studies demonstrated that liver stiffness (LS), as assessed by TE, was affected by food consumption from the mechanism of postprandial hyperemia [11-13]. High body mass index (BMI), alcohol drinking, histological steatosis grade 3 and $\mathrm{CAP}>323 \mathrm{~dB} / \mathrm{m}$ interfere with the accuracy of CAP measurement [14]. The effect of a meal on CAP has never been investigated. We aimed to evaluate the effect of a meal on CAP and LS. We hypothesized that CAP value might increase after food ingestion similar to the phenomenon of the meal effect on LS.

\section{Methods}

\section{Patients}

Patients with chronic liver diseases who underwent a percutaneous liver biopsy at the liver clinics of Ramathibodi Hospital between January 2015 and January 2016 were recruited. A liver biopsy was performed to identify the cause of abnormal liver tests or for staging of chronic liver diseases. Inclusion criteria were: (1) age $>18$ years old (2) interpretable liver biopsies including the length of the liver $>10 \mathrm{~mm}$. Exclusion criteria were: (1) contraindicated to TE (e.g. having congestive heart failure, pregnancy, ascites, hepatitis and/or cholestasis jaundice (aspartate aminotransferase $(\mathrm{AST})$ or alanine aminotransferase $(\mathrm{ALT})>$ five times upper normal, total bilirubin $>5 \mathrm{mg} / \mathrm{dl}$ ), (2) TE failure (interquartile range/median was more than 0.3 , a success rate lower than 60\%), (3) regular alcoholic drinking (>20 g/day), (4) end-stage renal disease, (5) refusal to participate with the study. The study was conducted according to the principles of the Declaration of Helsinki (revision of Edinburgh, 2000). The study was reviewed and approved by the Committee on Human Right Related to Research Involving $\mathrm{Hu}$ man Subjects, Faculty of Medicine, Ramathibodi Hospital, Mahidol University (ID 11-57-22). Written informed consent was obtained before the beginning of the study.

\section{Clinical evaluation and biochemical data}

Patients underwent abdominal ultrasonography and anthropometric measurement including body mass index (BMI) (weight $(\mathrm{kg}) /$ height $\left(\mathrm{m}^{2}\right)$ ), waist circumference and hip circumference. Demographic data were obtained from medical records. Biochemical data including ALT, alkaline phosphatase (ALP), albumin, total bilirubin, fasting blood sugar (FBS), triglyceride (TG), high-density lipoprotein cholesterol (HDL), serum creatinine, were collected within 4 weeks of the liver biopsy.

\section{Liver biopsy}

Patients underwent ultrasound-guided percutaneous liver biopsy. Liver biopsy was performed within 4 weeks of the study recruitment. Liver pathology was evaluated by a single experienced hepatopathologist who did not have access to clinical data of the study patients. Liver histology was graded for fibrosis (F) and steatosis (S) staging using Metavir scoring (F0-F4) and steatosis staging (: S0, < 5\%, S1, 5-33\%; S2, 34-66\%; and $\mathrm{S} 3,>66 \%)[2,15]$.

\section{Ultrasonography}

Patients underwent abdominal ultrasonography (HITACHI, Japan) using a convex $3.5 \mathrm{MHz}$ probe for evaluation of fatty liver on the same day with TE. Fatty liver was evaluated based on the degree of liver brightness, the presence of sonographic contrast appearance between liver and kidney parenchyma, reduced vessel wall echogenicity, high posterior attenuation and poorly delineated diaphragm [16].

\section{Measurement of liver stiffness (LS) and controlled attenuation parameter (CAP)}

A single operator who had experiences of more than 1000 cases of TE measurement performed TE in this study. While the patients were lying on their back, the tip of a $3.5 \mathrm{MHz}$ standard $\mathrm{M}$ probe was placed on the skin of intercostal space over the right lobe of the liver. The liver stiffness was measured with transient elastography (FibroScan ${ }^{\oplus}$, Echosens, Paris, France). LS is expressed in kilopascal $(\mathrm{kPa})$. The only results accepted followed the standard protocol with 10 valid measurements and had success rates over $60 \%$ and an interquartile range (IQR)/ median of less than 0.3 [17].

CAP is a novel non-invasive parameter to assess the degree of steatosis by using a vibration-controlled transient 
elastography $\left(\mathrm{VCTE}^{\mathrm{ru}}\right)$ device based on the properties of ultrasonic signals acquired by TE. A total ultrasonic attenuation (go-and-return path) was estimated. CAP was evaluated using the same radiofrequency data and region of interest used for the assessment of LS. CAP values were expressed in $\mathrm{dB} / \mathrm{m}$, range $100-400 \mathrm{~dB} / \mathrm{m}$ [8]. CAP value was interpreted only when the associated LS value was valid.

LS and CAP measurement were obtained simultaneously after an eight-hour overnight fasting (baseline values) and $15,30,45,60,90$, and $120 \mathrm{~min}$ following the intake of a standard commercial formula $(400 \mathrm{ml}, 600$ kcal, protein: carbohydrates: fat - 15: 55: 30 (equivalent to a standard Thai meal)), and every 30 min until the LS and CAP values returned to baseline.

\section{Statistical analysis}

For descriptive analysis, continuous variables were expressed as mean \pm standard deviation (SD) or median (Interquartile range - IQR), and categorical variables as number (\%). The multilevel mixed model approach was used to analyze the effect of a meal on CAP and LS at each time point compared to the baseline (or premeal values). We controlled for BMI and time by including both variables in the mixed linear models of CAP and LS. $P<0.05$ was taken as statistical significance. Statistical analysis was performed with Stata software version 14 .

\section{Results}

\section{Characteristics of patients}

Seventy-five patients underwent liver biopsy during the study period. Thirty-five were excluded due to the following reasons: end-stage renal disease (11), markedly elevated ALT or bilirubin (5), TE failure (2) or refusal to participate in the study (17). A total of 40 patients were enrolled to the study. Baseline characteristics and biochemical data are shown in Table 1 . The majority of study patients $(28 / 40,70 \%)$ were female. The mean age was $53.1 \pm 11.2$ years old. Chronic hepatitis B was the most common cause of liver disease (12/40, 30\%). Regarding the staging of liver fibrosis, there were 17 patients (42.5\%), 9 (22.5\%), $4(10.0 \%), 8(20.0 \%)$, and 2 (5.0\%) with F0, F1, F2, F3, and F4 stages, respectively (Table 2). According to steatosis staging, 22 (55.0\%), 11 (27.5\%), $4(10.0 \%)$ and $3(7.5 \%)$ of the patients had S0, S1, S2 and S3 stages, respectively. The mean (SD) CAP and median (range) LS at baseline were $249.7 \pm 58.1 \mathrm{~dB} / \mathrm{m}$ and $11.9(6-18.1) \mathrm{kPa}$.

\section{The effect of a meal on CAP and LS}

CAP and LS values were assessed after overnight fasting (baseline values) and at 15, 30, 45, 60, 90, $120 \mathrm{~min}$ following the intake of a standard commercial formula, and every $30 \mathrm{~min}$ until CAP and LS values returned to
Table 1 Demographic and laboratory data

\begin{tabular}{|c|c|}
\hline Characteristics & Patients $(n=40)$ \\
\hline Female $^{a}$ & $28(70.0 \%)$ \\
\hline Age, year ${ }^{b}$ & $53.1 \pm 11.2$ \\
\hline $\mathrm{BMl}, \mathrm{kg} / \mathrm{m}^{2 \mathrm{~b}}$ & $25.6 \pm 4.5$ \\
\hline Waist circumference, $\mathrm{cm}^{\mathrm{b}}$ & $88.4 \pm 11.9$ \\
\hline Hip circumference, $\mathrm{cm}^{\mathrm{b}}$ & $97.9 \pm 8.1$ \\
\hline Chronic Hepatitis $B^{a}$ & $12(30.0)$ \\
\hline Chronic Hepatitis $C^{a}$ & $5(12.5)$ \\
\hline NAFLD ${ }^{a}$ & $8(20)$ \\
\hline Other ${ }^{a}$ & $15(37.5)$ \\
\hline \multicolumn{2}{|l|}{ Biochemical parameters } \\
\hline AST, U/L ${ }^{b}$ & $71.9 \pm 35.6$ \\
\hline$A L T, U / L^{c}$ & $92.5(59.5-153.5)$ \\
\hline $\mathrm{FBS}, \mathrm{mg} / \mathrm{dl}^{\mathrm{b}}$ & $113.2 \pm 41.4$ \\
\hline Triglyceride, mg/dl ${ }^{b}$ & $117.7 \pm 65.5$ \\
\hline $\mathrm{HDL}, \mathrm{mg} / \mathrm{dl}^{\mathrm{b}}$ & $50.4 \pm 18.3$ \\
\hline
\end{tabular}

$S D$ standard deviation, NAFLD nonalcoholic fatty liver disease, BMI body mass index, AST aspartate aminotransferase, $A L T$ alanine aminotransferase, ALP alkaline phosphatase, FBS fasting blood glucose, HDL high density lipoprotein anumber (\%)

${ }^{\mathrm{b}}$ mean $\pm \mathrm{SD}$

'median (IQR)

baseline. A significant decline of CAP values following meal intake was observed 15 to $120 \mathrm{~min}(P<0.05)$ in Fig. 1. The CAP post-meal peak value was seen at 60 min $(P<0.01)$. All post-meal CAP values returned to baseline by $150 \mathrm{~min}$. The peak post-meal delta decrease of $\mathrm{CAP}$ was $18.1 \mathrm{~dB} / \mathrm{m}(P<0.01)$. A significant increase

Table 2 Radiological and histological data

\begin{tabular}{|c|c|}
\hline Characteristics & Patients $(n=40)$ \\
\hline \multicolumn{2}{|l|}{ Liver biopsy } \\
\hline \multicolumn{2}{|l|}{ Fibrosis stage } \\
\hline $\mathrm{F} 0 / \mathrm{F} 1 / \mathrm{F} 2 / \mathrm{F} 3 / \mathrm{F}^{\mathrm{a}}$ & $17(42.5) / 9(22.5) / 4(10.0) / 8(20.0) / 2(5)$ \\
\hline \multicolumn{2}{|l|}{ Steatosis stage } \\
\hline $\mathrm{S} 0 / \mathrm{S} 1 / \mathrm{S} 2 / \mathrm{S} 3^{\mathrm{a}}$ & $22(55.0) / 11(27.5) / 4(10.0) / 3(7.5)$ \\
\hline \multicolumn{2}{|c|}{ Transient elastography (Premeal) } \\
\hline$C A P, d B / m^{b}$ & $249.7 \pm 58.1$ \\
\hline $\mathrm{LS}, \mathrm{kPa}^{\mathrm{c}}$ & $11.9(6-18.1)$ \\
\hline \multicolumn{2}{|l|}{ Ultrasonography } \\
\hline No fatty liver ${ }^{a}$ & $17(46)$ \\
\hline Mild fatty liver ${ }^{a}$ & $14(37.8)$ \\
\hline Moderate fatty liver ${ }^{a}$ & $6(16.2)$ \\
\hline
\end{tabular}

Abbreviations: $S D$ standard deviation, NAFLD nonalcoholic fatty liver disease, $B M I$ body mass index, AST aspartate aminotransferase, ALT alanine aminotransferase, $A L P$ alkaline phosphatase, $F B S$ fasting blood glucose, $H D L$ high density lipoprotein, CAP controlled attenuation parameter, LSM liver stiffness

anumber (\%)

${ }^{b}$ mean $\pm S D$

'median (IQR) 


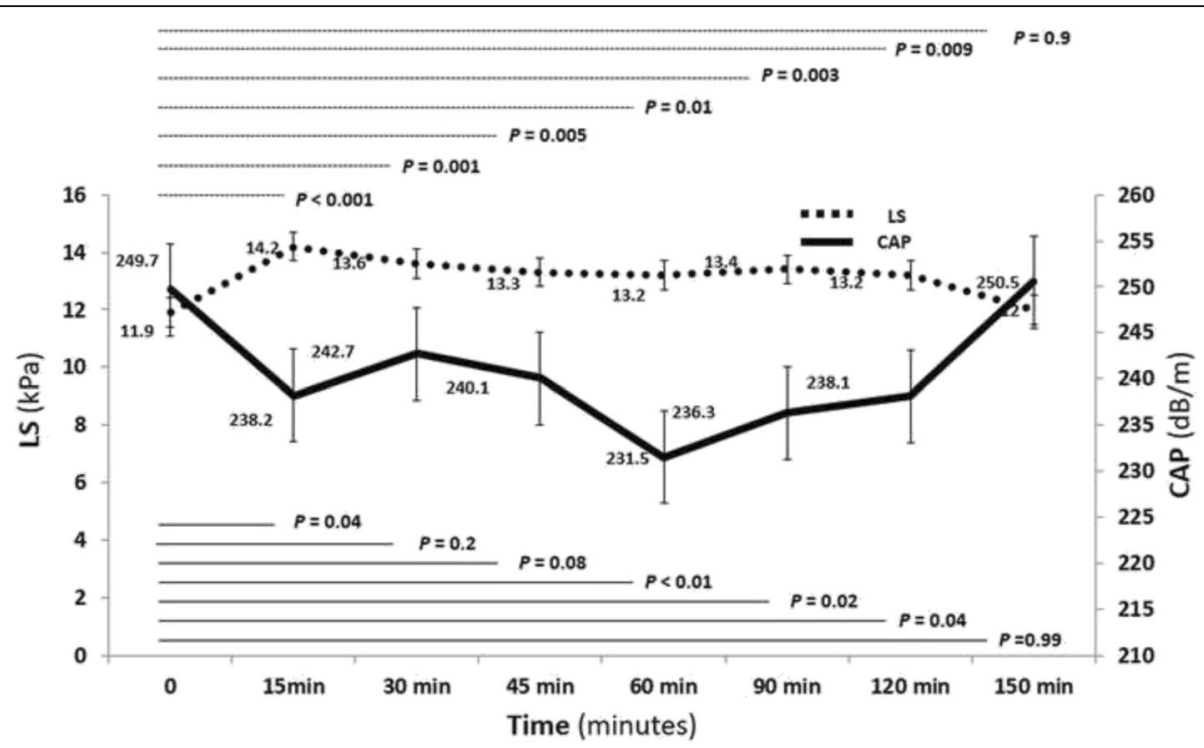

Fig. 1 Controlled attenuation parameter (CAP) and liver stiffness (LS) after meal intake

in LS values following meal intake was observed 15 to 120 min $(P<0.01)$, with the LS post-meal peak value at 15 min $(P<0.001)$. Post-meal LS values returned to baseline by $150 \mathrm{~min}$ in all. The peak post-meal delta increase of LS was $2.4 \mathrm{kPa}(P=0.001)$.

\section{The effect of meal on CAP according to fibrosis and steatosis stages}

For thirty (75\%) patients with F0-F2 stage, the mean CAP values following meal intake began to decline from $15 \mathrm{~min}(P=0.02)$ and peaked at $60 \mathrm{~min}(P=0.002)$ (Fig. 2a). The peak post-meal delta decrease of CAP values was $20.3 \mathrm{~dB} / \mathrm{m} \quad(P=0.002)$. The $C A P$ values returned to baseline by $90 \mathrm{~min}$. There were $10(25 \%)$ patients who had F3-F4 fibrosis stage (Fig. 2b). The mean CAP values following meal intake did not show significant change.

Thirty-three (82.5\%) patients had S0-S1 steatosis stage. The mean CAP values following meal intake was significant decline at $60 \mathrm{~min}(P=0.003)$ and at $90 \mathrm{~min}(P=$ 0.03 ), respectively (Fig. 3a). The mean post-meal CAP peak value was at $60 \mathrm{~min}$. The mean CAP values returned to baseline by $120 \mathrm{~min}$. There were 7 (17.5\%) patients who had S2-S3 steatosis stage. The mean CAP values following meal intake declined 15 to $120 \mathrm{~min}(P<0.05)$ (Fig. 3b). The mean post-meal CAP peak value was at $15 \mathrm{~min}$ and the CAP values returned to baseline by $150 \mathrm{~min}$.
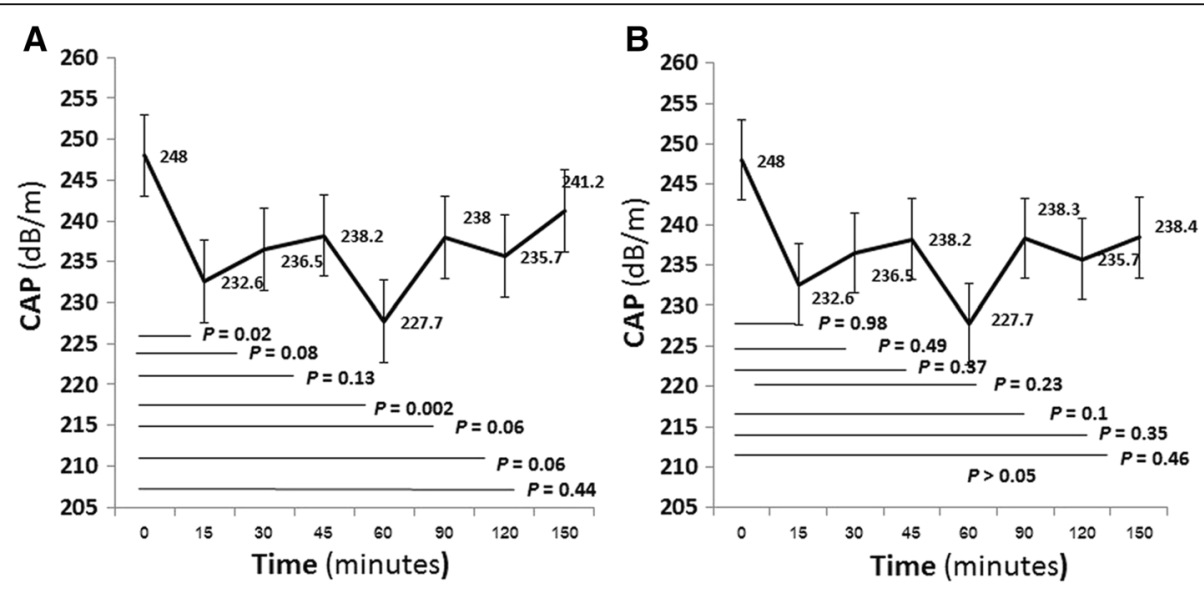

Fig. 2 Controlled attenuation parameter (CAP) after meal intake stratified by Metavir stage of liver fibrosis. a F0-F2. b F3-F4 

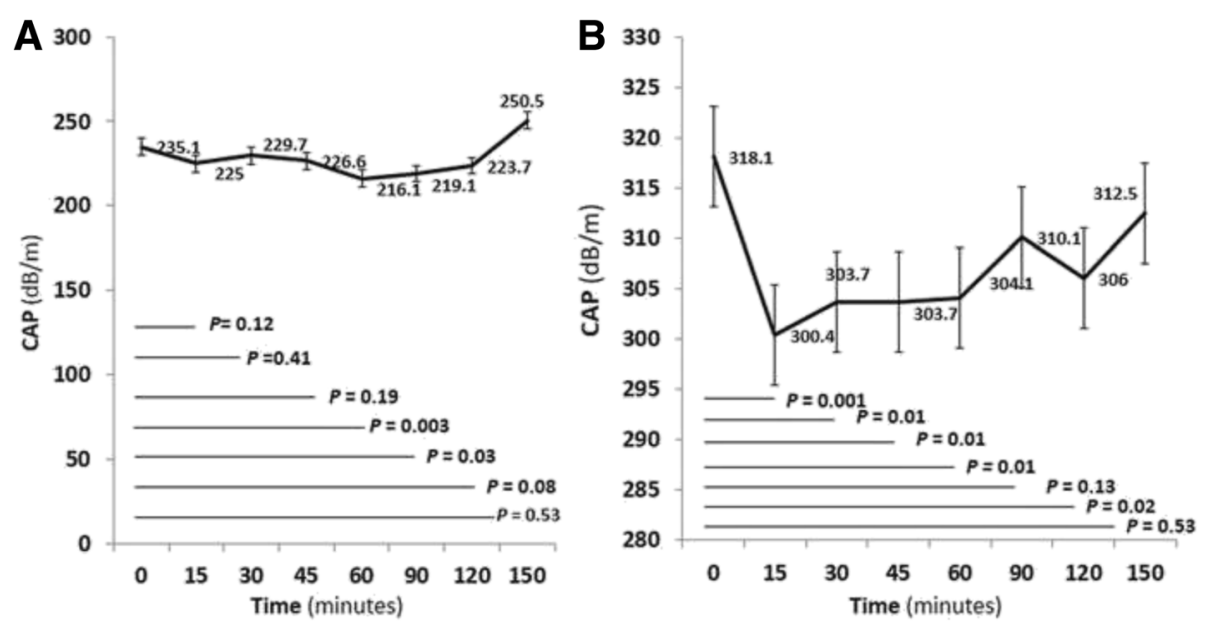

Fig. 3 Controlled attenuation parameter (CAP) after meal intake stratified by steatosis stage. a S0-S1. b S2-S3

\section{The effect of meal on LS according to fibrosis and steatosis stages}

Thirty (75\%) patients had F0-2 fibrosis stage, the mean LS values following meal intake was significantly increase at $15 \mathrm{~min}$ to $120 \mathrm{~min}$ (Fig. 4a). The peak of increment was at $15 \mathrm{~min}$. The mean LS values returned to baseline at $150 \mathrm{~min}$. There were $10(25 \%)$ patients who had F3-4 fibrosis stage. The mean LS values following meal intake showed significantly increase at 15 and $120 \mathrm{~min}(P=0.01$ and $P=0.003)$ (Fig. 4b). The peak of increment was at $120 \mathrm{~min}$. The mean LS values returned to baseline at $150 \mathrm{~min}$.

Thirty-three (82.5\%) patients had S0-S1 steatosis stage. The mean LS values following meal intake showed significantly increase at 15 to $120 \mathrm{~min}$ (Fig. 5a). The peak of increment was at $15 \mathrm{~min}$. The mean LS values returned to baseline at $150 \mathrm{~min}$. There were 7 (17.5\%) patients who had S2-S3 steatosis stage. The mean LS values following meal intake did not show any change over the time (Fig. 5b).

\section{Discussion}

We recruited 40 patients who had liver biopsy to the study. Contrary to our primary assumption, the CAP values were significantly reduced after meal intake $15 \mathrm{~min}$ to $120 \mathrm{~min}$, and the values returned to the premeal level by $150 \mathrm{~min}$. The CAP post-meal peak was observed at $60 \mathrm{~min}$ after meal intake. We found the same effect of meal intake on CAP values at early stages of fibrosis and across all stages of steatosis. The effect of meal on CAP values in the opposite direction with the effect of meal on LS values was showed in our study. The rising of LS values after meal in this study was similar to previous reports $[11-13,18]$. Undergoing transient
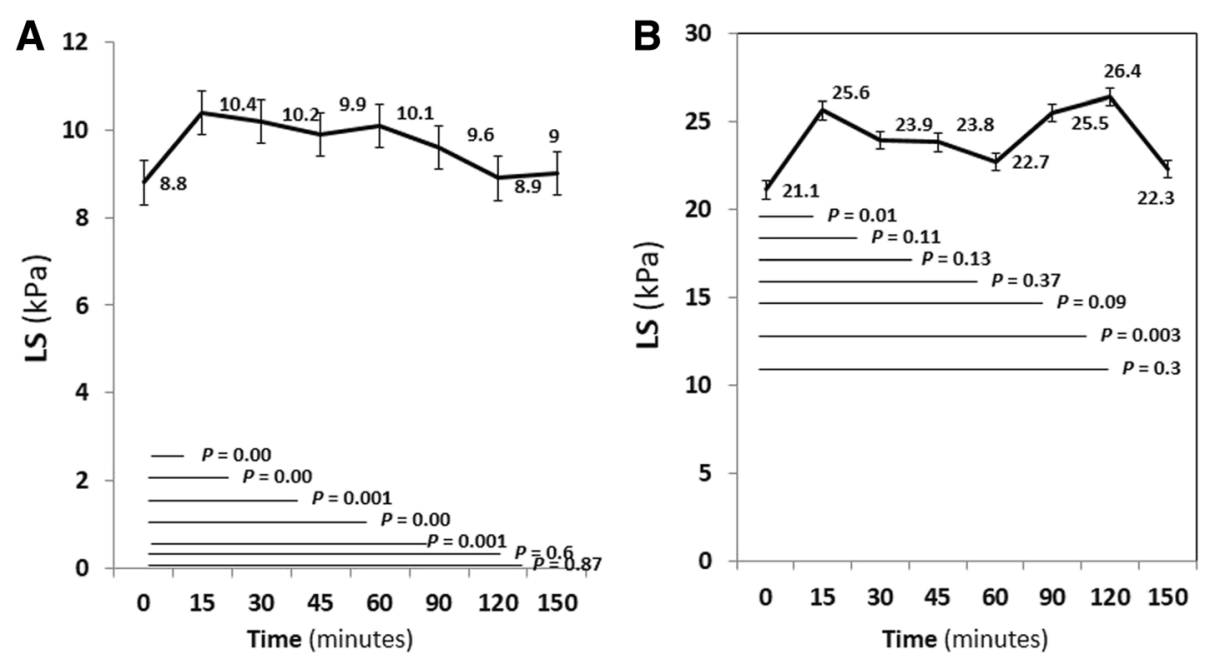

Fig. 4 Liver stiffness (LS) after meal intake stratified by Metavir stage of liver fibrosis. a F0-F2. b F3-F4 

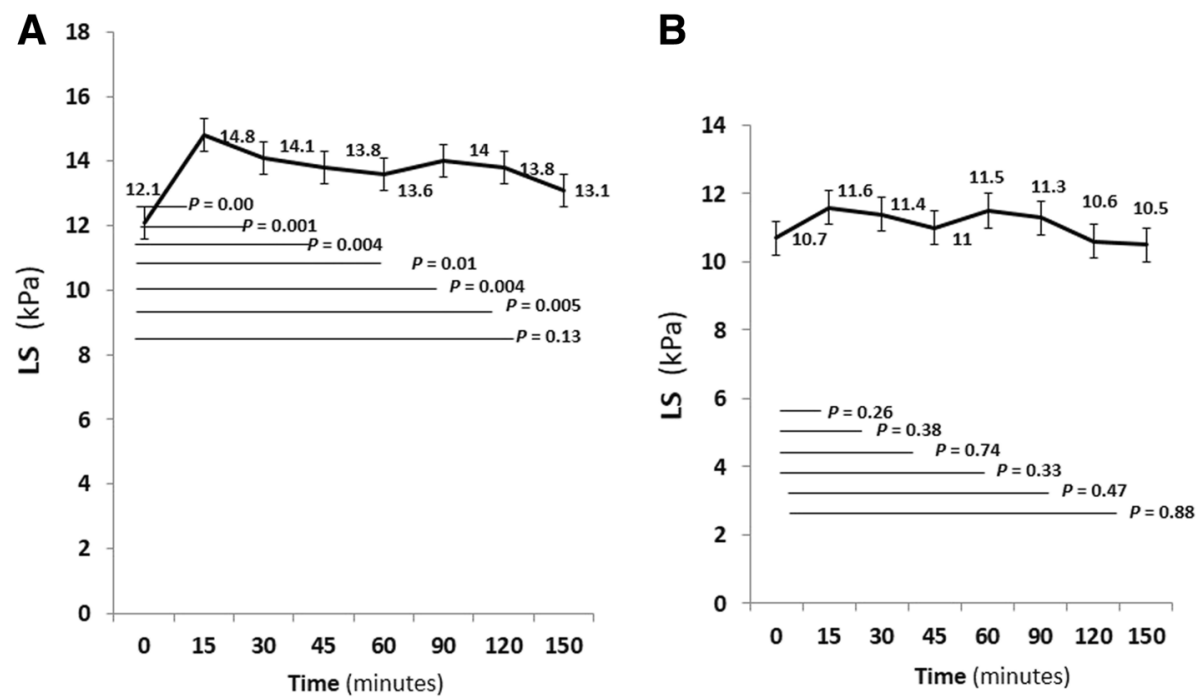

Fig. 5 Liver stiffness (LS) after meal intake stratified by steatosis stage. a S0-S1. b S2-S3

elastography without adequate fasting can lead to falsely decreased CAP value of $-18.1 \mathrm{~dB} / \mathrm{m}$ and incorrectly increased LS value of $+2.4 \mathrm{kPa}$ in average. LS is calculated from velocity. Velocity is related to tissue stiffness, the harder the tissue, the faster the shear wave propagation. However, for CAP, it measures the ultrasound attenuation. So, post-prandial hyperemia, LS increased while CAP decreased from reducing attenuation [19]. Due to the fact that $70 \%$ of study patients were female and $30 \%$ of liver disease were chronic hepatitis $\mathrm{B}$ and the mean BMI of subjects was $25.6 \pm 4.5 \mathrm{~kg} / \mathrm{m}^{2}$, the generalizability of the results study should be cautious. The accuracy of CAP measurement for steatosis determination is low in the patients with obesity and advanced fibrosis [20]. Furthermore, we did not have a control group with normal water taking to exclude the influence of liquid volume as in the study of Mederacke I, et al. [11].

The LS values were significantly elevated after meal intake 15 to $120 \mathrm{~min}$, and they returned to baseline by $150 \mathrm{~min}$. The LS post-meal peak value was observed at 15 min. However, the changing of LS values after meal in our study had somewhat differences from previous reports in term of the returning time to baseline level $[11,12]$. An elevation of postprandial LS value was observed 15 to 45 min after meal intake, with returning to baseline level within $120 \mathrm{~min}$ in that study [12]. Because only patients with chronic hepatitis $\mathrm{C}$ were included [12], the difference in the etiology of liver diseases may explain some dissimilar findings. Furthermore, from the study by Mederacke et al., a standardized continental breakfast, which consisted of two rolls, ham, cheese, butter and jam with approximately $600 \mathrm{kcal}, 54 \%$ carbohydrates, $26 \%$ fat and $20 \%$ protein, an elevation of LS values followed meal intake occurred 15 to $60 \mathrm{~min}$, and returned to baseline at $180 \mathrm{~min}$ [11]. Although the meal in our study and that of Mederacke et al. was similar in the amount of energy and the proportion of nutrients, the returning time of LSM values to baseline in their study was $30 \mathrm{~min}$ longer. This phenomenon may be explained by the different types of diet in the two studies. Liquid meal was chosen in our study instead of solid meal. Moreover, LS values at $150 \mathrm{~min}$ were not performed in that study [11]. To the best of our knowledge, our report is the first experimental study to explore the effect of meal on CAP in addition to LS values.

There is some limitation in our study. The protocol was not designed to explain the mechanism that resulted in the changing of CAP and LS values following meal intake. Furthermore, it was conducted with a small number of patients and as a single center study. This topic requires further research in a larger population to confirm our findings.

\section{Conclusions}

CAP values declined after meal intake with the postmeal peak value at $60 \mathrm{~min}$, contrasting with the rising of LS values after meal intake with the post-meal peak value at $15 \mathrm{~min}$. TE performed without adequate fasting can yield a falsely lower value of CAP and misleadingly higher value of LS. A fasting period of more than $150 \mathrm{~min}$ after a meal is recommended for patients undergoing TE for measuring LS and CAP.

\section{Abbreviations}

ALP: Alkaline phosphatase; ALT: Alanine aminotransferase; AST: Aspartate aminotransferase; BMI: Body mass index; CAP: Controlled attenuation parameter; F: Fibrosis; FBS: Fasting blood sugar; HDL: High density lipoprotein cholesterol; IQR: Interquartile range; LS: Liver stiffness; NAFLD: Nonalcoholic fatty liver disease; S: Steatosis; TE: Transient elastography; TG: Triglyceride 


\section{Acknowledgements}

Not applicable.

\section{Funding}

This study was funded by Faculty of Medicine, Ramathibodi Hospital, Mahidol University and the Gastroenterological Association of Thailand. They did not have roles in the design of the study and collection, analysis, interpretation of data or writing of the manuscript.

\section{Availability of data and materials}

The dataset used and/or analyzed during the current study is available from the corresponding author on reasonable request.

\section{Authors' contributions}

KR participated in the design of the study, data collection, interpreted the data, and drafted the manuscript. SR contributed to the design of the study, interpreted and analyzed the data and reviewed the manuscript. KP and PS participated in the the design of the study, data collection and involved in drafting the manuscript. AS participated in the design of the study, interpreted the data, drafted and edited the manuscript. All authors gave final approval of the final version of the manuscript to be published, and all are agree to be accountable to all aspects of the work.

\section{Competing interests}

The authors declare that they have no competing interest.

\section{Consent for publication}

Not applicable.

\section{Ethics approval and consent to participate}

The study protocol was approved by the Committee on Human Rights related to Research Involving Human Subjects, Faculty of Medicine, Ramathibodi Hospital (ID 11-57-22). It was carried out according to the Good Clinical Practice Guideline after obtaining informed consent. Informed consent obtained from the study participants as written as indicated in the methods section.

\section{Publisher's Note}

Springer Nature remains neutral with regard to jurisdictional claims in published maps and institutional affiliations.

\section{Author details}

'Division of Gastroenterology and Hepatology, Department of Medicine, Faculty of Medicine, Ramathibodi Hospital, Mahidol University, 270 Rama 6 Road, Bangkok 10400, Thailand. ${ }^{2}$ Section for Clinical Epidemiology and Biostatistics, Faculty of Medicine, Ramathibodi Hospital, Mahidol University, Bangkok 10400, Thailand.

Received: 20 December 2016 Accepted: 3 April 2017

Published online: 13 April 2017

\section{References}

1. Angulo P. Nonalcoholic fatty liver disease. N Engl J Med. 2002;346:1221-31.

2. Kleiner DE, Brunt EM, Van Natta M, Behling C, Contos MJ, Cummings OW, et al. Design and validation of a histological scoring system for nonalcoholic fatty liver disease. Hepatology. 2005;41:1313-21.

3. Leandro G, Mangia A, Hui J, Fabris P, Rubbia-Brandt L, Colloredo G, et al. Relationship between steatosis, inflammation, and fibrosis in chronic hepatitis C: a meta-analysis of individual patient data. Gastroenterology. 2006;130:1636-42.

4. Poynard T, Ratziu V, McHutchison J, Manns M, Goodman Z, Zeuzem S, et al. Effect of treatment with peginterferon or interferon alfa-2b and ribavirin on steatosis in patients infected with hepatitis C. Hepatology. 2003;38:75-85.

5. Rockey DC, Caldwell SH, Goodman ZD, Nelson RC, Smith AD. Liver biopsy. Hepatology. 2009;49:1017-44.

6. Myers RP, Fong A, Shaheen AA. Utilization rates, complications and costs of percutaneous liver biopsy: a population-based study including 4275 biopsies. Liver Int. 2008;28:705-12

7. Ratziu V, Charlotte F, Heurtier A, Gombert S, Giral P, Bruckert E, et al. Sampling variability of liver biopsy in nonalcoholic fatty liver disease. Gastroenterology. 2005;128:1898-906.
8. Sasso M, Beaugrand M, de Ledinghen V, Douvin C, Marcellin P, Poupon R, et al. Controlled attenuation parameter (CAP): a novel VCTE quided ultrasonic attenuation measurement for the evaluation of hepatic steatosis: preliminary study and validation in a cohort of patients with chronic liver disease from various causes. Ultrasound Med Biol. 2010;36:1825-35.

9. de Ledinghen V, Vergniol J, Foucher J, Merrouche W, le Bail B. Non-invasive diagnosis of liver steatosis using controlled attenuation parameter (CAP) and transient elastography. Liver Int. 2012;32:911-8.

10. Sasso M, Tengher-Barna I, Ziol M, Miette V, Fournier C, Sandrin L, et al. Novel controlled attenuation parameter for noninvasive assessment of steatosis using Fibroscan: validation in chronic hepatitis C. J Viral Hepat. 2012;19:244-53.

11. Mederacke I, Wursthorn K, Kirschner J, Rifai K, Manns MP, Wedemeyer H, et al. Food intake increases liver stiffness in patients with chronic or resolved hepatitis C virus infection. Liver Int. 2009;29:1500-6.

12. Arena U, Lupsor Platon M, Stasi C, Moscarella S, Assarat A, Bedogni G, et al. Liver stiffness is influenced by a standardized meal in patients with chronic hepatitis $C$ virus at different stages of fibrotic evolution. Hepatology. 2013;58:65-72

13. Berzigotti A, De Gottardi A, Vukotic R, Siramolpiwat S, Abraldes JG, GarciaPagan JC, et al. Effect of meal ingestion on liver stiffness in patients with cirrhosis and portal hypertension. PLoS One. 2013;8:e58742.

14. Jung KS, Kim BK, Kim SU, Chon YE, Chun KH, Kim SB, et al. Factors affecting the accuracy of controlled attenuation parameter (CAP) in assessing hepatic steatosis in patients with chronic liver disease. PLoS One. 2014:9:e98689.

15. Brunt EM, Janney CG, Di Bisceglie AM, Neuschwander-Tetri BA, Bacon BR. Nonalcoholic steatohepatitis: a proposal for grading and staging the histological lesions. Am J Gastroenterol. 1999;94:2467-74.

16. Hamer OW, Aguirre DA, Casola G, Lavine JE, Woenckhaus M, Sirlin CB. Fatty liver: imaging patterns and pitfalls. Radiographics. 2006;26:1637-53.

17. de Ledinghen V, Vergniol J, Capdepont M, Chermak F, Hiriart JB, Cassinotto $\mathrm{C}$, et al. Controlled attenuation parameter (CAP) for the diagnosis of steatosis: a prospective study of 5323 examinations. J Hepatol. 2014;60:1026-31.

18. Tangpradabkiet W, Chamroonkul N, Vitheerungroj T, Piratvisuth T. Influence of food intake on measurement of liver stiffness by transient elastography in patients with chronic viral hepatitis B and C. Thai J Gastroenterol. 2013;14:16-22.

19. Yoshioka K, Hashimoto S, Kawabe N. Measurement of liver stiffness as a non-invasive method for diagnosis of non-alcoholic fatty liver disease. Hepatol Res. 2015:45:142-51.

20. Fujimori N, Tanaka N, Shibata S, Sano K, Yamazaki T, Sekiguchi T, et al. Controlled attenuation parameter is correlated with actual hepatic fat content in patients with non-alcoholic fatty liver disease with none-to-mild obesity and liver fibrosis. Hepatol Res. 2016:46:1019-27.

\section{Submit your next manuscript to BioMed Central and we will help you at every step:}

- We accept pre-submission inquiries

- Our selector tool helps you to find the most relevant journal

- We provide round the clock customer support

- Convenient online submission

- Thorough peer review

- Inclusion in PubMed and all major indexing services

- Maximum visibility for your research

Submit your manuscript at www.biomedcentral.com/submit
Biomed Central 